\title{
Effect of private insurance incentive policy reforms on trends in coronary revascularisation procedures in the private and public health sectors in Western Australia: a cohort study
}

\author{
Shauna Trafalski ${ }^{1}$, Tom Briffa ${ }^{2}$, Joseph Hung ${ }^{3}$, Rachael E Moorin ${ }^{4}$, Frank Sanfilippo ${ }^{2}$, David B Preen ${ }^{1}$
} and Kristjana Einarsdóttir ${ }^{*}$

\begin{abstract}
Background: The Australian federal government introduced private health insurance incentive policy reforms in 2000 that increased the uptake of private health insurance in Australia. There is currently a lack of evidence on the effect of the policy reforms on access to cardiovascular interventions in public and private hospitals in Australia. The aim was to investigate whether the increased private health insurance uptake influenced trends in emergency and elective coronary artery revascularisation procedures (CARPs) for private and public patients.

Methods: We included 34,423 incident CARPs from Western Australia during 1995-2008 in this study. Rates of emergency and elective CARPs were stratified for publicly and privately funded patients. The average annual percent change (AAPC) in trend was calculated before and after 2000 using joinpoint regression.

Results: The rate of emergency CARPs, which were predominantly percutaneous coronary interventions (PCIs) with stenting, increased throughout the study period for both public and private patients (AAPC $=12.9 \%, 95 \% \mathrm{Cl}=5.0,22.0$ and $14.1 \%, 95 \% \mathrm{Cl}=9.8,18.6$, respectively) with no significant difference in trends before and after policy implementation. The rate of elective PCls with stenting from 2000 onwards remained relatively stable for public patients (AAPC $=-6.0,95 \% C=-16.9,6.4)$, but increased by $4.1 \%$ on average annually $(95 \% \mathrm{Cl}=1.8,6.3)$ for private patients ( $p_{\text {difference }}=0.04$ between groups). This rate increase for private patients was only seen in people aged over 65 years and people residing in high socioeconomic areas.

Conclusions: The private health insurance incentive policy reforms are a likely contributing factor in the shift in 2000 from public to privately-funded elective PCls with stenting. These reforms as well as the increasing number of private hospitals may have been successful in increasing the availability of publicly-funded beds since 2000 .
\end{abstract}

Keywords: Health insurance, Coronary artery disease, Revascularisation procedures, Health policy

\section{Background}

The Australian federal government introduced the private health insurance incentive policy reforms in 19972000 to encourage the uptake of private health insurance and increase the availability of beds in public hospitals [1,2]. From 1984, Australian residents had been able to

\footnotetext{
* Correspondence: keinarsdottir@ichr.uwa.edu.au

${ }^{5}$ Telethon Institute for Child Health Research, Centre for Child Health Research, The University of Western Australia, 100 Roberts Road, Subiaco 6008, Western Australia

Full list of author information is available at the end of the article
}

access free treatment in public hospitals covered by national health insurance (public patients) and as a result, private health insurance membership among the Australian population began to decline [3]. These policy reforms were introduced as a response to this declining trend so that more patients would choose to be treated as private patients in either private or public hospitals at a subsidised cost through private health insurance $[4,5]$.

The policy reforms were efficient in increasing private health insurance uptake, demonstrated by a rise in the percentage of population with private health insurance 
from $30 \%$ in 1999 to $45 \%$ in 2001 [6]. Some evidence suggests that the reforms increased private health insurance utilisation as well, particularly for surgical in-patients episodes $[7,8]$. However, there is currently a lack of empirical evidence on the effect of the policy reforms on cardiovascular interventions and whether the reforms were successful in relieving pressure on access to coronary interventions in public hospitals. Such information is particularly important as cardiovascular diseases are the most expensive group of diseases in Australia [9]. Coronary heart disease (CHD) alone accounted for $31 \%$ of the total cardiovascular disease cost in $2004-5$ or $\$ 1.8$ billion [9], with almost threequarters of the cost due to CHD spent on hospitaladmitted patients [9]. This high cost of cardiovascular interventions in hospitals can mainly be attributed to coronary artery revascularisation procedures (CARPs) as they are the primary procedural intervention for stable and unstable CHD in Australia. These procedures include coronary artery bypass grafting (CABG) and percutaneous coronary intervention (PCI), with or without stenting [10].

The high investment in private health insurance by the Australian federal government and the high economic burden of CARPs on the health care system, emphasises the need to investigate this important issue. The objective of this study was therefore to assess whether the increased uptake of private health insurance in Australia after the implementation of the Federal government's policy initiatives (around the year 2000) resulted in more CARPs being performed in the private sector and if so, what impact it had on CARPs in the public sector and whether trend were consistent across age and socioeconomic status (SES).

\section{Methods}

\section{Study design}

In this study, rates of emergency and elective CARPs were calculated from 1 January 1995 to 31 December 2008 in Western Australia (WA). The rates were stratified for publicly and privately funded patients.

The procedure rates were calculated from the yearly procedure counts in our data (numerators) and annual population figures for $35+$ year old individuals resident in WA (denominators) based on 5-yearly census data published by the Australian Bureau of Statistics [11]. When we analysed elective PCIs with stenting by age and SES group, both numerators and denominators were stratified according to the respective groups.

\section{Study sample}

All CARPs in WA were identified using routinely-collected administrative health data from the WA Hospital Morbidity Data Collection at the Department of Health. The data fields included up to 11 procedures, 21 diagnoses, dates and types of admissions (emergency or elective) and separations for all inpatient episodes in all WA public and private hospitals.

The study cohort included all individuals aged 35 years and over who had their index (or first) CARP at a WA hospital between 1 January 1995 and 31 December 2008. Hospital admissions dating back to 1 January 1985 were available to determine prior CARPs using the International Classification of Disease (ICD) codes for the procedures, versions 9 and 10, as shown in Table 1. First-ever CARP was defined on the basis of no coronary revascularization procedure recorded on a WA hospital admission in the previous 10 years. CARPs performed on an elective admission (elective CARPs) were distinguished from emergency CARPs, defined as a CARP performed during a nonelective (emergency) cardiovascular admission.

The hospital data also contained information on the Index of Relative Socio-Economic Disadvantage (IRSD) [12] and the funding source (private or public) of the patient at the time of their CARP. The IRSD values are based on information on household income, educational attainment and occupation from the Australian Census conducted every five years and assigned to each collection district area, about 250 households, in the state. The IRSD values were divided into two groups with those living in the lowest 40\% SES areas classified as low SES with others classified as living in a high SES area. Patient funding source was binary; those who elected to be treated as public patients and those who elected to be treated as private patients, independent of whether the hospital where they underwent treatment was classed as a public or private institution. Public patients consisted of those who were insured under the Australian Health Care Agreement and Reciprocal Health Care Agreements. Private patients included those who were funded through private health insurance or were self-funded.

\section{Statistical analysis}

The average annual percent change (AAPC) and 95\% confidence intervals for the trend in procedure rate during 1995-2008 was calculated using the Joinpoint Regression Program Version 3.4.3 (http://surveillance. cancer.gov/joinpoint/). The joinpoint regression analysis was used to identify points at which statistically significant changes in temporal trend occurred and to calculate the annual percent change in each segment. The average percent change in procedure rate per year across the joinpoint segments was then calculated for the entire study period (1995-2008) as well as two separate time periods; $1995-2000$ and 2000-2008. A negative AAPC indicated a decreasing trend on average whereas a positive AAPC indicated an increasing trend on average. All other analyses were performed using the statistical software SAS version 9.3 (SAS Institute Inc., Cary, NC, USA). 
Table 1 CARPs ICD9 and 10-AM procedure codes

\begin{tabular}{lll}
\hline & ICD-9 and ICD-9-CM & ICD-10-AM \\
\hline CABG & $36.10-36.19$ & $38497,38500,38503,90201$ \\
PCI without stenting & $36.01,36.02,36.05$ & $35304-00,35305-00$, \\
& & $38303-00,38300-00$ \\
PCI with stenting & $36.06,36.07$ & $35310(-00,-01,-02)$, \\
& & $3830600-00,38306-01$, \\
& & $38306-02$ \\
\hline
\end{tabular}

\section{Results}

From January 1995 to December 2008 there were 34,423 index CARPs performed in WA hospitals, including 14,089 (41\%) classified as emergency CARPs and 20,334 (59\%) as elective. From the index CARP cases, $57.4 \%$ were publicly funded patients and $42.6 \%$ were privately funded, with $94 \%$ of the public patients treated in public hospitals and $72 \%$ of the private patients treated in private hospitals. Privately funded patients were $40 \%$ pre- 2000 , which increased to $44 \%$ post- 2000 .

The proportion of patients treated in public hospitals whether privately or publicly funded - was lower for pre-2000 than post-2000, whereas the proportion of private hospital patients increased regardless of their funding status (Table 2). This trend was particularly evident for patients undergoing elective CARPs as the proportion of public patients treated in public hospitals declined from 51.1\% in the period prior to year 2000 to $39.4 \%$ after year 2000 with the proportion of private patients treated in public hospitals showing a similar pattern in terms of absolute percent decrease (15.1\% to $5.7 \%)$.
The rate of all emergency CARPs increased steadily before and after the year 2000 (Figure 1a) for both public $(60 \%$ in 1995 to $84 \%$ in 2008$)$ and private patients (19\% to $40 \%)$. The rate of PCIs with stenting showed a similar escalating trend pattern to all CARPs (Figure 1b). The rate of emergency PCIs without stenting (Figure 1c) decreased before year 2000 similarly in both groups $\left(p_{\text {difference }}=0.6\right)$ and then remained relatively stable at a low rate from 2000 onwards. The rate of all emergency CABGs declined in public patients before and after 2000 (AAPC $=-5.8,95 \% \mathrm{CI}=-7.2,-4.4$ ) and remained relatively steady at a low rate throughout in private patients (AAPC $=0.2,95 \% \mathrm{CI}=-2.8,3.3$ ).

The rate of all elective CARPs decreased for both public and private patients during $1995-2008$ from $84 \%$ to $44 \%$ and $90 \%$ to $79 \%$ respectively (Figure $2 \mathrm{a}$ ). This decrease was similar between the two groups before 2000 ( $p_{\text {difference }}=0.9$ ), but from 2000 onwards the decrease attenuated for private patients $(\mathrm{p}<0.0001)$. The rate of elective PCIs with stenting increased for both public and private patients during 1995-2000 ( pdifference $=0.9$ ), but from 2000 onwards the rate increased only for private patients by $4.1 \%(95 \% \mathrm{CI}=1.8,6.3)$ on average annually, while it remained stable for public patients $(\mathrm{AAPC}=-6.0,95 \% \mathrm{C}=-16.9,6.4)\left(\mathrm{p}_{\text {difference }}=0.04\right)$. Elective PCIs without stenting decreased in both public and private patients before the year 2000, and thereafter remained steady at a low rate in both groups ( $\mathrm{p}_{\text {difference }}=0.6$ ). Elective CABGs decreased throughout the study period with no apparent differences between public and private patients.

Table 2 Proportion of index CARP patients $(n=34,423)$, elective $(n=20,334)$ and emergency $(n=14,089)$ by funding source, hospital type and time period

\begin{tabular}{|c|c|c|c|}
\hline & Before 2000 & After 2000 & Total \\
\hline All & N (\%) & N (\%) & N (\%) \\
\hline Public patient in public hospital & $6,363(58.9)$ & $12,275(52.0)$ & $18,638(54.1)$ \\
\hline Public patient in private hospital & $115(1.1)$ & $1,000(4.2)$ & $1,115(3.2)$ \\
\hline Private patient in public hospital & $1,696(15.7)$ & $2,422(10.3)$ & $4,118(12.0)$ \\
\hline Private patient in private hospital & $2,621(24.3)$ & 7,931 (33.6) & $10,552(30.7)$ \\
\hline \multicolumn{4}{|l|}{ Elective } \\
\hline Public patient in public hospital & $3,787(51.1)$ & $5,092(39.4)$ & $8,879(43.7)$ \\
\hline Public patient in private hospital & $59(0.8)$ & $550(4.3)$ & $609(3.0)$ \\
\hline Private patient in public hospital & $1,121(15.1)$ & $742(5.7)$ & $1,863(9.2)$ \\
\hline Private patient in private hospital & $2,441(33.0)$ & $6,541(50.6)$ & $8,983(44.2)$ \\
\hline \multicolumn{4}{|l|}{ Emergency } \\
\hline Public patient in public hospital & $2,576(76.1)$ & $7183(67.1)$ & $9,759(69.3)$ \\
\hline Public patient in private hospital & $56(1.7)$ & $450(4.2)$ & $506(3.4)$ \\
\hline Private patient in public hospital & $575(17.0)$ & $1680(15.7)$ & $2,255(16.0)$ \\
\hline Private patient in private hospital & $180(5.3)$ & $1389(13.0)$ & $1,569(11.1)$ \\
\hline
\end{tabular}

$\mathrm{CARP}=$ coronary artery revascularisation procedure. 


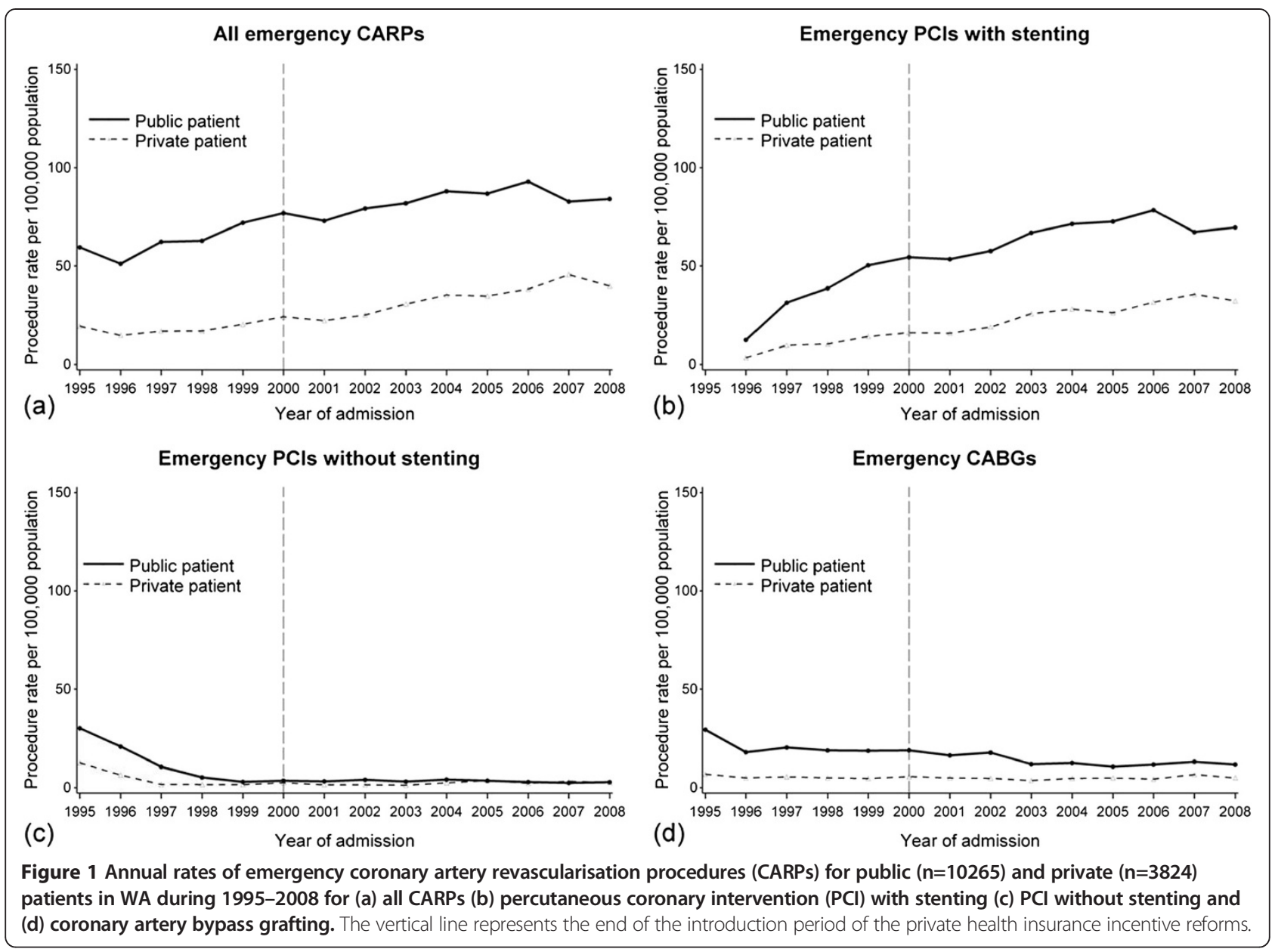

Figure 3 shows elective PCIs with stenting in public and private patients stratified by age 35-64 and 65+ years and by high and low SES. Post year 2000, the rate of elective PCIs with stenting in public patients remained relatively unchanged irrespective of age group and SES. For private patients post-2000, the rate of elective PCIs with stenting increased more steeply in people aged $65+$ years ( $\mathrm{AAPC}=7.2 \%, 95 \% \mathrm{CI}=2.9,11.5$ ) than their younger counterparts (AAPC $=1.7,95 \% \mathrm{CI}=-0.2,3.7$ ) as well as for private patients living in high SES areas (AAPC $=5.1 \%$, 95\% $\mathrm{CI}=-0.6,11.0$ ), compared with low $\mathrm{SES}$ areas (AAPC $=0.8 \%$, $95 \% \mathrm{CI}=-0.4,2.0)$.

\section{Discussion}

Our results describe the trend in the rate of emergency and elective CARPs in WA for public and private funding sources before and after the introduction of the Federal government's private health insurance incentive policy reforms in 2000. The rates of emergency procedures - which included mainly PCIs with stenting - increased throughout the study period for both public and private patients. Elective PCIs with stenting, however, increased only for privately funded patients following the introduction of the private health insurance incentive policy reforms. This rate increase was only evident for people aged $65+$ years and people living in high SES areas.

Despite the steady increase in emergency PCIs with stenting for both public and private patients throughout the study period, our results showed that the majority of these procedures continue to be performed in the public sector based on the absolute procedural rates. Over $80 \%$ of patients requiring emergency CARPs are still treated in public hospitals as a public or private patient. As such, there appears to be a core group of patients that will continue to require emergency CARPs, predominantly PCIs with stenting for management of acute coronary syndromes, and who are not influenced by private health insurance policy reforms to purchase private health. However, there was a minor impact in the sense that the proportion of emergency CARPs which were performed in private hospitals increased from $7 \%$ to $17.2 \%$ before and after year 2000. This increase in rate of emergency CARPs is likely to be attributed to many factors, including an ageing population, evidence-based practice of primary PCI for ST segment elevation myocardial infarction [13], 


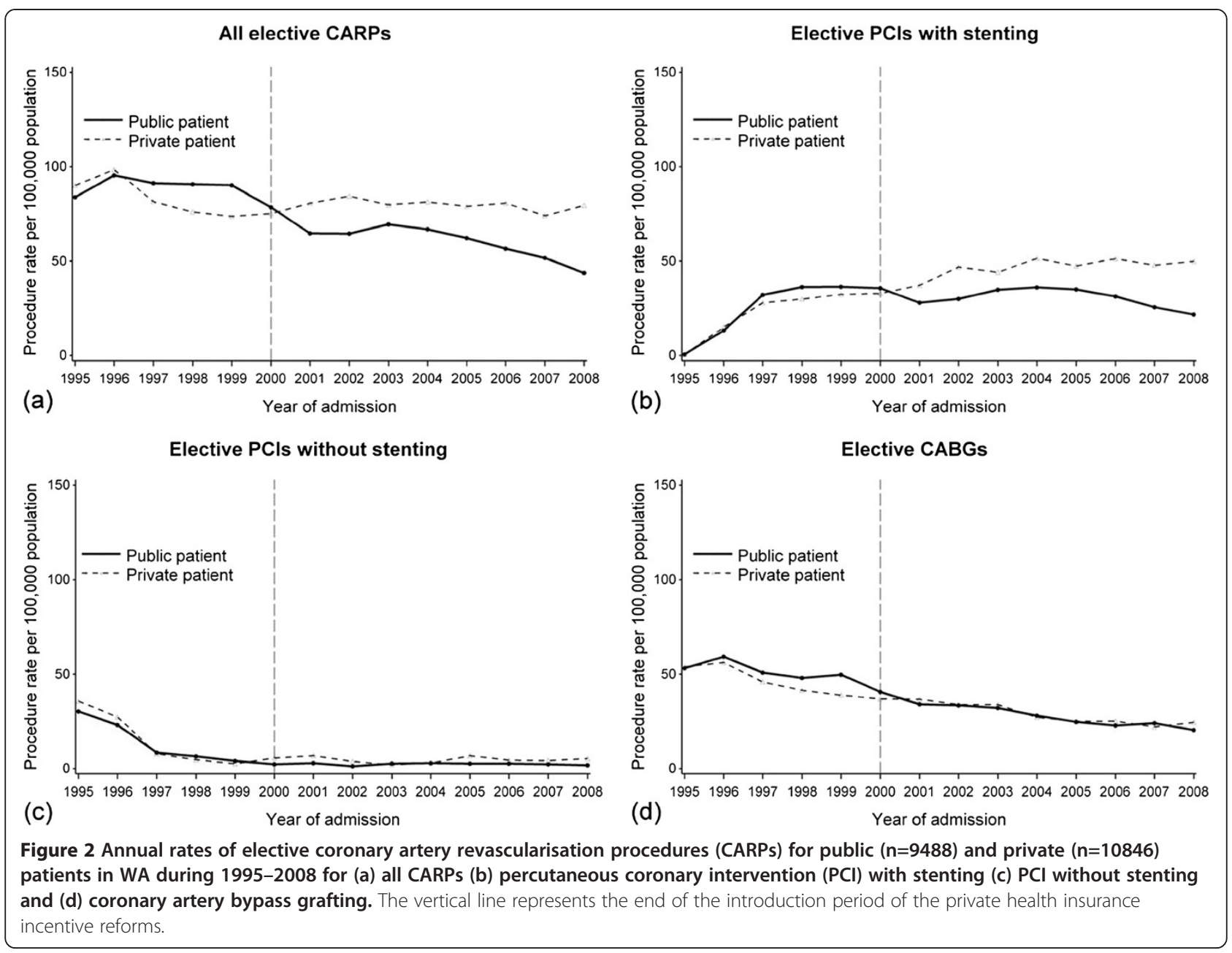

and current clinical guidelines that recommend an early invasive management strategy over an initially conservative strategy for acute coronary syndrome cases $[14,15]$. Our results also showed a declining trend for elective CABGs in both public and private patients. This predated the policy reforms and appears not to have been influenced by their introduction.

In our study, the rate of elective PCIs with stenting increased only for private patients from 2000 onwards. Notwithstanding controlling for increased availability of PCI since the introduction of the private health insurance incentive policy reforms, this initiative appears to have been successful in shifting elective PCIs with stenting from the public to the private sector particularly in the older age group which traditionally has been the group who are most disadvantaged in terms of access to elective surgery in public hospitals. Interestingly, the findings from this study also indicate that the increased rate of PCIs with stenting for private patients from 2000 onwards was only evident for people living in high SES areas. Therefore, it seems possible that mainly Western Australians with sufficient discretionary income were able to respond to the increased affordability of private health insurance membership following the introduction of the private health insurance policy reforms by shifting from the public to the private sector. The reason why there was not a comparable increase in rate of elective PCIs with stenting for private patients living in low SES areas is unclear because the rates of cardiovascular disease would be expected to be higher in people living in low SES areas and suggests that a person's SES and the area in which he/she lives has a significant effect on access to health care regardless of access to private health insurance.

The shift from publicly funded to privately funded elective PCIs with stenting from 2000 would likely have relieved the economic pressure on the public sector. However, due to the high cost of these procedures in the private sector and the increasing investment of the federal government towards the private sector, the shift may in fact not have improved the economic burden on the public purse. For example, the private health insurance incentives that were introduced in 2000 involved a $1 \%$ tax-penalty for high income earners without health insurance, a $30 \%$ tax rebate on insurance premiums and 


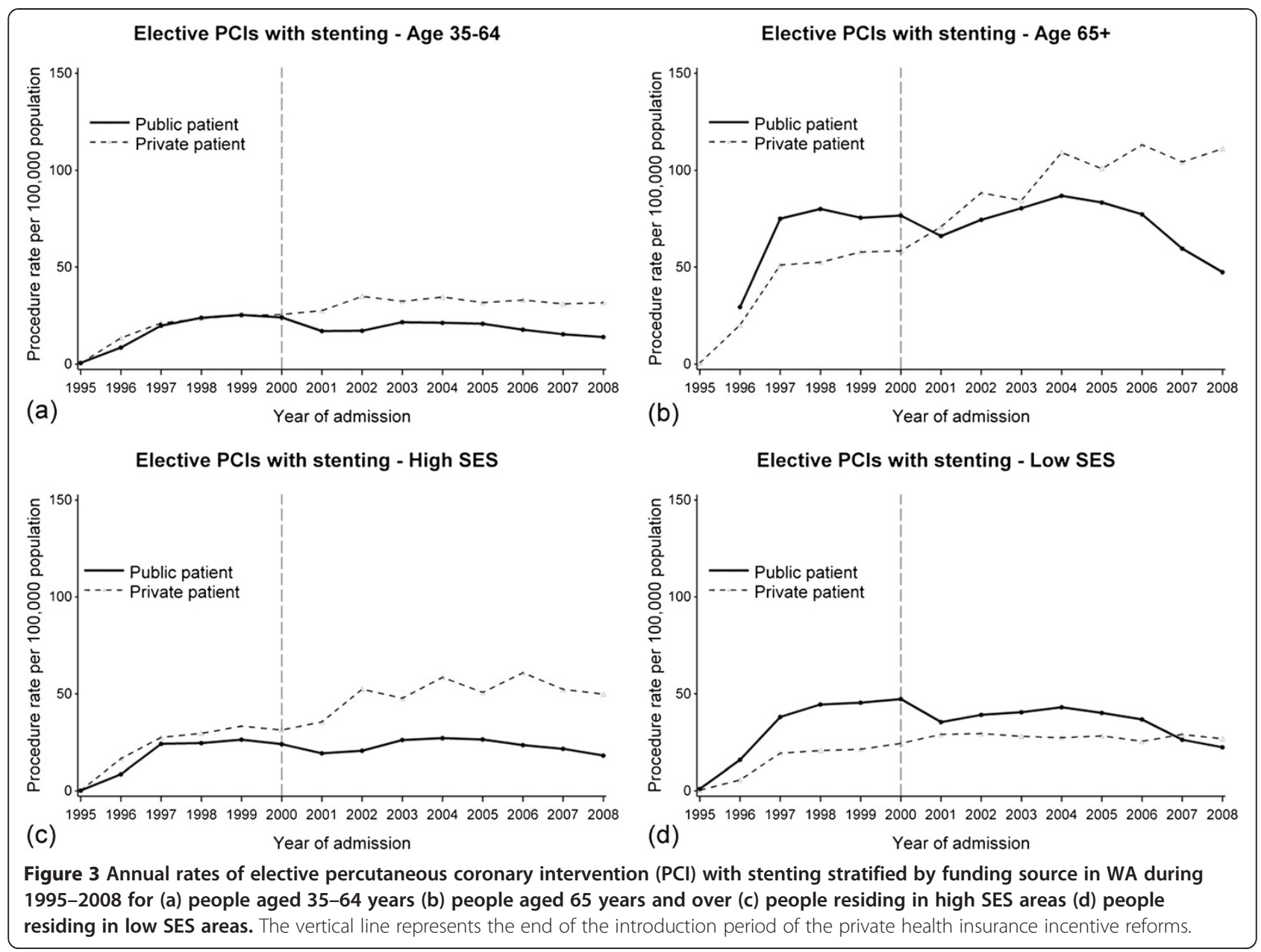

a $2 \%$ premium penalty pa for those who entered after the age of $30[1,16]$. The $50 \%$ increase in private health insurance membership following the introduction of these reforms has been attributed primarily to the introduction of the premium penalty as the $30 \%$ premium rebate was reported to increase private health insurance coverage by only $1 \%$ from 1998 to 1999 [17,18]. It therefore seems that the most costly policy reform, i.e. the $30 \%$ rebate, did not do the trick. However, because of the $30 \%$ rebate, government funding for patients undergoing invasive cardiac procedures (F42B) was lower for public patients ( $\$ 1,424$ per episode) than for private patients $(\$ 2,254)$ in 2005-6 [19]. In addition, since the premium rebate is $35 \%$ for people aged $65-69$ years and $40 \%$ for people aged 70 years and over, the government funded a greater proportion of private patient costs for the older age groups [19]. Furthermore, the costs of PCI procedures have been found to be more than twice as high in the private sector compared with the public sector [20]. As a result, although it has been argued that the policy reforms were successful in relieving economic pressure on the public sector, our results present some evidence to suggest that this may not necessarily have been the case.

What appears to be more likely than a reduction in the economic pressure on public hospitals is that the policy reforms may have increased the availability of beds in public hospitals. The vast majority of public patients included in this study were treated in public hospitals and the proportion of patients undergoing CARPs in public hospitals decreased significantly from pre-2000 to post-2000. This decreasing trend was particularly evident for elective procedures. This supports our results suggesting that when private health insurance became more affordable in the year 2000, it was the elective cases that tended to transfer to the private sector. What also seems to have made this shift easier is that the number of private hospitals in Australia had been increasing since the 1990. For example, there was a $16 \%$ increase in the number of private hospitals in Australia during 1990-2000, whereas public hospital numbers increased by only $3 \%$ over the same time [21].

The strength of this study is reflected in the use of 14 years of routinely-collected hospital inpatient information 
for the entire WA population. It is a statutory requirement that the Department of Health records information on all hospital admissions and separations from all public and private hospitals in the State. The hospital data collection has undergone stringent quality assessments by the Department of Health and validation studies have confirmed the accuracy of the coding [22,23]. Despite these strengths, some limitations require acknowledgement. Firstly, we were not able to quantify or account for the increase in catheter laboratories in metropolitan Perth or the increase in cardiologists who can perform PCIs with stenting. We can thus not say for certain whether the shift from publicly-funded elective PCIs with stenting to privately-funded elective PCIs with stenting around 2000 was due to the private health insurance incentive policy reforms alone. Secondly, we cannot distinguish between bare stents and drug eluting stents. This is a constraint since drug eluting stents were more commonly used in the private sector. They were also more expensive and delivered better near-term or early procedural outcomes compared with bare stents. And thirdly, our data showed that not all private patients were treated in private hospitals, a small proportion of the privately funded index CARP patients were treated in a public hospital.

\section{Conclusions}

This study investigated whether the increased uptake of private health insurance following the Australian private health insurance incentive policy reforms introduced in 2000 resulted in an increased rate of CARPs in patients electing for private care. Our results show a rise of privately funded elective PCIs with stenting after 2000, but a decline in publicly funded elective procedures. As such, these reforms may have been successful in improving access to elective revascularization procedures overall and reducing pressure on public hospitals for the interventional management of acute coronary syndromes in Western Australia. However, due to the high cost of these procedures in the private sector and the increasing investment of the federal government towards the private sector, the shift may not have improved the economic burden on the public purse.

\section{Abbreviations \\ AAPC: Average annual percent change; PHI: Private health insurance; CARP: Coronary artery revascularisation procedure; WA: Western Australia; HMDC: Hospital morbidity data collection; CABG: Coronary artery bypass grafting; PCl: Percutaneous coronary intervention; CHD: Coronary heart disease; IRSD: Index of relative socio-economic disadvantage.}

\section{Competing interests}

The authors declare that they have no competing interests.

\section{Authors' contributions}

ST analysed the data and wrote the article. TB and JH supervised the work and gave clinical advice on cardiovascular disease. RM, FS and DBP gave statistical input and advice on Australian health care policy and analysis of administrative data. KE initiated the research, designed the study, and interpreted the data. All authors revised the paper critically for important intellectual content and approved it to be published.

\section{Acknowledgements}

The authors wish to thank the staff at the Western Australian Data Linkage Branch, and the Department of Health Inpatient Data Collections and Registrar General for the provision of data. This work was funded by a Grant-In-Aid from the Heart Foundation of Australia (G 11P 5661).

\section{Author details}

${ }^{1}$ Centre for Health Services Research, School of Population Health, The University of Western Australia, 35 Stirling Highway, Crawley, Perth 6009, Western Australia. ${ }^{2}$ Cardiovascular Research Group, School of Population Health, The University of Western Australia, 35 Stirling Highway, Crawley, Perth 6009, Western Australia. ${ }^{3}$ School of Medicine and Pharmacology, Sir Charles Gairdner Hospital Unit and The University of Western Australia, 35 Stirling Highway, Crawley, Perth 6009, Western Australia. ${ }^{4}$ Centre for Population Health Research, Curtin Health Innovation Research Institute, Curtin University, Kent Street, Bentley, Perth 6102, Western Australia.

${ }^{5}$ Telethon Institute for Child Health Research, Centre for Child Health Research, The University of Western Australia, 100 Roberts Road, Subiaco 6008, Western Australia.

Received: 4 April 2013 Accepted: 18 July 2013

Published: 22 July 2013

\section{References}

1. Butler JR: Policy change and private health insurance: did the cheapest policy do the trick? Aust Health Rev 2002, 25(6):33-41.

2. Segal $L$ : Why it is time to review the role of private health insurance in Australia. Aust Heal Rev 2004, 27:3-15.

3. Private Health Insurance Administration Council (PHIAC). Membership Statistics. http://www.phiac.gov.au/for-industry/industry-statistics/.

4. Foster M, Fleming J: The Health Care System in Australia. In Health Care Practice in Australia. Melbourne, Australia: Oxford University Press; 2008:46-73.

5. Harris MG, Harris RD: The Australian health system: continuity and change. J Health Hum Serv Adm 1998, 20(4):442-467.

6. Cormack M: Private health insurance: the problem child faces adulthood. Aust Health Rev 2002, 25(2):38-51.

7. Moorin RE, Brameld KJ, Holman CD: Health care financing and public responses: use of private insurance in Western Australia during 1980-2001. Aust Heal Rev 2006, 30(1):73.

8. Moorin RE, Holman CDAJ: Do marginal changes in PHI membership accurately predict marginal changes in $\mathrm{PHI}$ use in Western Australia? Health Policy 2006, 76(3):288-298.

9. Health care expenditure on cardiovascular diseases 2004-05. Canberra: Australian Institute of Health and Welfare, Canberra: Cat. no. CVD 43; 2008.

10. Patel MR, Dehmer GJ, Hirshfeld JW, Smith PK, Spertus JA: ACCF/SCAI/STS/ AATS/AHA/ASNC/HFSA/SCCT 2012 Appropriate Use Criteria for Coronary Revascularization Focused Update. J Am Coll Cardiol 2012, 59(9):857-881.

11. Population by Age and Sex, Australian States and Territories. Canberra: Australian Bureau of Statistics, Canberra: cat. no. 3201.0; 2011.

12. Australian Bureau of Statistics: Information Paper. An introduction to socio-economic indexes for areas (SEIFA), Australia 2006. In ABS Cat No 20390. Canberra, Australia: ABS; 2008.

13. Steg PG, James SK, Atar D, Badano LP, Lundqvist CB, Borger MA, Di Mario C, Dickstein K, Ducrocq G, Fernandez-Aviles F, et al: ESC Guidelines for the management of acute myocardial infarction in patients presenting with ST-segment elevation. Eur Hear J 2012, 33(20):2569-2619.

14. Wright RS, Anderson JL, Adams CD, Bridges CR, Casey JDE, Ettinger SM, Fesmire FM, Ganiats TG, Jneid H, Lincoff AM, et al: 2011 ACCF/AHA Focused Update of the Guidelines for the Management of Patients With Unstable Angina/ Non-ST-Elevation Myocardial Infarction (Updating the 2007 Guideline) A Report of the American College of Cardiology Foundation/American Heart Association Task Force on Practice Guidelines Developed in Collaboration With the American College of Emergency Physicians, Society for Cardiovascular Angiography and Interventions, and Society of Thoracic Surgeons. J Am Coll Cardiol 2011, 57(19):1920-1959.

15. Chew DP, Amerena J, Coverdale S, Rankin J, Astley C, Brieger D: Current management of acute coronary syndromes in Australia: observations from the acute coronary syndromes prospective audit. IMJ 2007. 37(11):741-748.

16. Segal L: Why it is time to review the role of private health insurance in Australia. Aust Health Rev 2004, 27(1):3-15. 
17. Palangkaraya A, Yong J, Webster E, Dawkins P: The income distributive implications of recent private health insurance policy reforms in Australia. Eur J Health Econ 2009, 10(2):135-148.

18. Walker AE, Percival R, Thurecht $L$, Pearse J: Public policy and private health insurance: distributional impact on public and private hospital usage. Aust Health Rev 2007, 31(2):305-314.

19. Funding sources for admitted patients in Australian hospitals, 2005-06. Canberra: Australian Institute of Health and Welfare, Canberra: Cat. no. HWE 44; 2009.

20. Harper RW, et al: Costs, charges and revenues of elective coronary angioplasty and stenting: the public versus the private system. Med J Aust 2000, 173(6):296-300.

21. Australian hospital statistics 2000-01. Canberra: Australian Institute of Health and Welfare, Canberra: AlHW cat. no. HSE 20; 2002.

22. Teng T-HK, Finn J, Hung J, Geelhoed E, Hobbs M: A validation study: how effective is the Hospital Morbidity Data as a surveillance tool for heart failure in Western Australia? Aust N Z J Public Health 2008, 32(5):405-407.

23. Crome M: Monitoring the incidence of cardiovascular disease in Australia. In AIHW Cat No CVD 16 (Cardiovascular Disease Series No 17). Canberra, Australia: AlHW; 2001.

doi:10.1186/1472-6963-13-280

Cite this article as: Trafalski et al.: Effect of private insurance incentive policy reforms on trends in coronary revascularisation procedures in the private and public health sectors in Western Australia: a cohort study. BMC Health Services Research 2013 13:280.

\section{Submit your next manuscript to BioMed Central and take full advantage of:}

- Convenient online submission

- Thorough peer review

- No space constraints or color figure charges

- Immediate publication on acceptance

- Inclusion in PubMed, CAS, Scopus and Google Scholar

- Research which is freely available for redistribution 